Published in final edited form as:

Curr Opin Cardiol. 2019 May ; 34(3): 241-245. doi:10.1097/HCO.0000000000000615.

\title{
Noncoding RNAs in cardiovascular diseases
}

\author{
Priyatansh Gurha \\ Center for Cardiovascular Genetics, Institute of Molecular Medicine and Department of Medicine, \\ University of Texas Health Sciences Center at Houston, and Texas Heart Institute, Houston, TX \\ 77030., Tel: +1 713-500-2335, Priyatansh.gurha@uth.tmc.edu
}

\section{Abstract \\ Purpose of review: Human genome is pervasively transcribed, producing coding and noncoding RNAs. Recent studies have revealed the roles of a class of noncoding RNAs, the long noncoding RNAs (lncRNAs), in heart failure and other cardiovascular diseases. This review provides a brief summary of recent findings on lncRNA function.}

Recent findings: Recent studies have documented the roles of lncRNAs in cardiac regeneration, conduction, hypertrophy/dysfunction, and endothelial function. LncRNAs perform these functions through acting as competing RNA (by binding and sequestering miRNAs) or acting as guides to protein targeting. A few lncRNAs also encode small peptides (for example; Dworf RNA) and in the context of heart regulate cardiac calcium homeostasis.

Summary: Noncoding RNA provides a versatile mechanism of gene regulation and thereby present as novel targets for intervention in various cardiovascular disease. Future studies aimed at defining the context-dependent IncRNA mechanisms will be required to advance our understanding and relish the goal of RNA therapeutics.

\section{Keywords}

Heart failure; LncRNA; miRNA and Cardiac regeneration

\section{Introduction}

Despite significant improvements, heart failure remains a leading health epidemic [1]. Therefore, identification and characterization of promising new avenues to explore for risk mitigation in $\mathrm{HF}$ are essential. The human genome is extensively transcribed, resulting in the production of numerous noncoding RNAs (ncRNAs) [2-4]. The ncRNAs are generally classified based on size as small ( $<200$ nucleotides, such as miRNA, piRNA, snoRNAs, etc.) and long noncoding RNAs (IncRNAs), which are generally defined as transcripts larger than 200 bases [4-9]. LncRNAs comprise a diverse class of RNAs, namely long intergenic RNA (lincRNA), enhancer RNAs (eRNAs), and sense or antisense transcripts (AS) [9, 10]. They are often expressed in a high cell- and tissue-specific manner and have been proposed to carry out varied functions, including transcriptional regulation in cis or trans, nuclear

Conflicts of interest

There are no conflicts of interest. 
domain formation/ organization, regulation of protein function and stability, and at times encoding for small proteins [5, 6, 8, 11-17]. Likewise, a subset of IncRNAs are produced from enhancer or promoter regions and likely regulate transcriptional activity from these regulatory elements. Abnormalities of lncRNAs function and expression have been directly linked with cardiovascular biology and disease. The roles of lncRNAs in cardiovascular disorders are beginning to emerge; of particular interest is the dysregulation of lncRNA regulatory circuits in cell fate, cardiac hypertrophy, vascular disease, atherosclerosis, and metabolic syndrome [5, 6, 8, 11-17]. In light of these discoveries, in this review, we provide information on some of the recent findings and highlight the role of lncRNAs in cardiac regeneration, cardiac arrhythmia, cell death, and endothelial biology. We also summarize new findings on a class of non-conventional lncRNAs that code for the small peptide (micro peptide) and regulate cardiac muscle function.

\section{LncRNAs in Cardiac regeneration}

The neonatal heart has regenerative capacity driven largely thorough cardiac myocyte proliferation, the adult heart, on the other hand, has limited regeneration capacity $[18,19]$. The mechanisms of this post-mitotic cell cycle exit, associated with cessation in cardiac proliferation and regeneration of myocardium, are not clearly defined. Several new studies have implicated lncRNAs in cardiac myocyte proliferation and thereby ushered in the new field of cardiac regeneration. A brief summary of these findings is presented here.

a) CAREL: A lncRNA named cardiac regeneration-related long noncoding ribonucleic acid (CAREL or NONMMUT070401) was found to be induced in neonatal mice upon cessation of cardiac myocyte proliferation [20]**. Gain of function studies using CARELTransgenic mice showed its ability to suppress cardiac proliferation as evidenced by increased $\mathrm{pH}^{+}$and Aurora $\mathrm{B}^{+}$myocyte. Furthermore, in the context of myocardial infarction (MI), silencing of CAREL improved heart function associated with increased cell proliferation and regeneration in neonatal and adult mice. Mechanistically, CAREL was proposed to act as a competing RNA for miR-296 and overexpression of miR-296, mimics CAREL loss of function phenotype. Targeting of Trp53inp1 and Itm2a by miR-296 was proposed to be a plausible mechanism of action. Thus, the findings implicate CAREL in the suppression of cardiomyocyte cell proliferation and induction of cell cycle exit through inhibition of miR-296.

b) CRRL (cardiomyocyte regeneration-related IncRNA): Like CAREL another long noncoding RNA namely CRRL (cardiomyocyte regeneration-related lncRNA) was shown to be a negative regulator of cardiomyocyte proliferation and cardiac repair [21] *. CRRL was identified to be induced in adult as compared to fetal cardiac tissues. Suppression of CRRL in a rat model of MI improved cardiac function and promoted cardiomyocyte proliferation and repair. CRRL exerts these effects through binding to miR-199a-3p and thereby suppressing its activity and increasing levels of its target Норx. The latter is implicated in the regulation of cardiac gene expression and cardiac development [22].

c) AZIN2-sv/NONHSAT002258: AZIN2-sv, a non-coding splice variant of the Azin2 gene, was identified as a potential cardiac lncRNA that was highly expressed in adult heart, 
and conserved in rodents [23] *. In vitro and in vivo inhibition of Azin2-svin cardiomyocytes enhanced proliferation rate, whereas its overexpression significantly reduced proliferation. The mechanism of action of Azin2-sv in cardiomyocytes was mediated through the miRNA-214/PTEN/Akt axis. Specifically, Azin2-sv acted as a miR-214 sponge thereby depressing PTEN expression. Furthermore, stabilizing of PTEN was further reinforced by the direct binding of Azin2-sV to PTEN and enhancing proliferation.

Similar to the above-mentioned lncRNAs several others have been shown to mediate cardiomyocyte proliferation mainly by perturbing miRNAs and direct binding to proteins $[12,24-26] *$.

Even though the initial studies described here have provided the impetus for defining the role of IncRNA in cardiac regeneration, future studies are required to rigorously define (by integrating multiple biochemical, molecular approaches) the role of lncRNAs on proliferation and maturation of cardiac myocyte. Likewise, careful studies designed to determine the effect of postnatal cardiac myocyte proliferation on cardiac hypertrophy and failure are required to ascertain the impact of lncRNAs on myocardial regeneration.

\section{LncRNAs in cardiac conduction system:}

Several ncRNAs (mostly miRNA) have been shown to regulate cardiac rhythm. Recently cardiac conduction regulatory $\underline{R} N A$ (CCRR) was identified as an antiarrhythmic lncRNA [27]**. CCRR is downregulated in failing human and mouse hearts. CCCR downregulation was associated with slow cardiac conduction and enhanced arrhythmogenicity in mice. CCRR overexpression rescues these detrimental effects. The biological role of CCRR is manifested through its interaction with a protein CIP85 whereby the CCRR-CIP85 complex occludes CIP85 mediated degradation of gap junction protein CX43. Knockdown or downregulation of CCRR causes perturbation of cell-cell junction integrity (intercalated discs and gap junctions) thorough degradation of CX43 by CIP85 interaction.

Downregulation of CX43 mediates electrical uncoupling and thereby increased the propensity to cardiac arrhythmias. Even though these studies do not exclude mechanism other than IncRNAs in the maintenance and alteration of Intercalated disc (ID) and Gap junction, CCRR still provides a potential therapeutic avenue for targeting pathological arrhythmias.

\section{LncRNAs in cardiac hypertrophy and dysfunction:}

Maladaptive cardiac remodeling due to sustained cardiac hypertrophy leads to decreased compliance and increased risk for heart failure. Several signaling pathways are known to contribute to the pathogenesis of pathological hypertrophy, and heart failure, a subset of those are driven by $\mathrm{Ca}^{2+}$ dysregulation [28, 29]. Here lncRNAs that are regulating these pathways and their role in cardiac function is described.

a) LncRNA ZFAS1: Long noncoding RNA ZFAS1 (ZNFX1 antisense1) is produced from a snoRNA host gene. In MI mouse models, Zfas1 was shown to be induced mainly in the cytoplasm and sarcoplasmic reticulum[30, 31]**. Overexpression of $Z$ fas 1 in mice 
decreased while its knockdown rescued contractile dysfunction in the context of MI. Zfas 1 binds SERCA2A protein and impairs its activity, leading to altered $\mathrm{Ca}^{2+}$ transient and intracellular $\mathrm{Ca}^{2+}$ overload in cardiomyocytes. Finally, only a small conserved sequence domain of $Z$ fas 1 gene is required and sufficient for its function. Given the targeting of SERCA2A in HF, ZFAS1 provides another opportunity for modulating SERCA2A activity and cardiac function in $\mathrm{HF}$

b) Airn: Several long noncoding RNAs cluster with imprinted genes, of these antisense Igf2r $\underline{R} N A$ noncoding antisense (Airn), has been extensively studied. The Airn regulates expression of $I g f 2 r$ and $S / c 22 a 2$ and $S / c 22 a 3$ genes either through transcriptional interference or through epigenetic silencing [32]. However, several isoforms of Airn are known to exist. Cardiac-specific Airn Isoform (Airn-O01) is shown to regulate cell death [33]. Further detailed mechanistic studies showed direct binding of Airn-01 to IGF2BP2 protein. This RNP complex is required for IGF2BP2 translation and also for the translation of several proteins that are targeted by IGF2BP2.Thus, Airn regulates cardiac translational output through IGF2BP2 and thereby cell survival.

\section{LncRNAs regulating endothelial function:}

Recent studies have implicated a crucial role of lncRNAs in in vascular biology. Several IncRNAs with a role in vascular remodeling, endothelial cell proliferation, angiogenesis, and cholesterol metabolism have been described $[6,8,34]$. Here we highlight two recent studies characterizing the role of IncRNAs in macrophage and BMC in the pathogenesis of artheoscloris.

a) MeXis: The liver X receptor (LXR) is a member of the nuclear receptor family of transcription factors that regulate cholesterol homeostasis and is implicated in the pathogenesis of atherosclerosis [35]. Activation of LXRs promotes reverse cholesterol transport in part through plasma membrane transporter ABCA1. LncRNA MeXis (Macrophage-expressed LXR-induced sequence) was shown to be induced upon LXR treatment [36]**. This induction of MeXis was correlated with the activation of its neighboring gene $A b c a 1$. MeXis regulates $A b c a 1$ expression through remodeling the local chromatin accessibility and direct recruitment of transcriptional coactivator DDX17. MeXis loss of function (LOF) in mice leads to decreased $A b c a 1$ expression, and impaired cholesterol efflux leading to atherosclerosis. MeXis human homolog TCONS00016111 was also found to regulate ABCA1 expression. Therefore, lncRNA MeXis-ABCA1 provide a novel regulatory target involved in the pathogenesis of atherosclerosis.

b) MALAT1: MALAT1 (Metastasis Associated Lung Adenocarcinoma Transcript 1) is a nuclear-enriched long coding RNA that lack PolyA tail and was initially implicated in cancer metastasis and cell migration. Recent studies have highlighted its role in the heart where it is involved in cardiac fibrosis, stress remodeling, and angiogenesis. However, its exact role in the endothelial function and the pathogenesis of atherosclerosis was not known. Using Apoe $^{-/-}$and Malat $^{-/-}$mice, the role of Malat 1 in plaque size and infiltration of $\mathrm{CD} 45^{+}$cells was revealed. Bone marrow transplantation experiments using Malat $1^{-/-}$bone marrow cells in $\mathrm{Apoe}^{-/-}$mice further confirmed its role in atherosclerotic lesion formation 
and proatherosclerotic phenotype [37]*. Malat1 was found to act as a sponge for miR-503. MALAT1 expression also correlated with enhanced human plaques and worse prognosis.

LncRNA coding for micropeptide: Recent genome-wide studies provide credence to the hypothesis that lncRNAs can also serve as a reservoir for coding functional micro peptides [38-40].The micro peptide discovery has been hindered largely because of the difficulty in identifying functional small open reading frames (ORFs). Regardless of this limitation, several micro peptides that affect the cardiovascular system has been identified. Of note are the conserved peptide myoregulin and DWORF (DWarf Open Reading Frame) both affecting SERCA (sarcoendoplasmic reticulum calcium transport ATPase) activity [41, 42]. However, the molecular underpinning of micro peptide function and their relevance to heart failure was not demonstrated.

Recently using DWORF-KO mice investigator have now determined that the effect of DWORF is mediated through its high-affinity binding to SERCA2A [43]**. This highaffinity binding is sufficient to compete with a SERCA2A inhibitor phospholamban (PLN) and restore SERCA2A activity and $\mathrm{Ca}_{2}{ }^{+}$transient in PLN-Tg mice. Furthermore, the relevance of DWORF to human HF was shown using a dilated cardiomyopathy mouse model of muscle-specific LIM domain protein knockout (MLP-KO). These mice show severe cardiac dilation, dysfunction and reduced SERCA2A activity. Overexpression of DWORF through a transgenic approach led to the rescue of cardiac function that was associated with increased SERCA2A activity and enhanced calcium transition. Thus, DWORF as a potent activator of SERCA2A within the heart is an attractive candidate for a heart failure therapy.

\section{Conclusion}

Here, the latest reports describing the role of lncRNAs in cardiovascular biology and disease are reviewed. Although several more lncRNAs are shown associated with cardiac development and disease, their functionality and therapeutic utility remain to be determined. More mechanistic studies that employ genome-wide tools with specific subcellular localization and classic RNA-protein interaction studies will be required to define the actual mode of lncRNA function. Likewise, more work is needed to determine whether lncRNAs could be used as biomarkers for cardiovascular diseases. The restricted spatiotemporal expression of lncRNAs provides a novel tool to treat cardiac cell type-specific lncRNAs without fortuitous effect on other cells and organs. Finally, novel tools for IncRNA cargo delivery have to be developed for effective treatment. In conclusion, these initial studies and future mechanistic details will enhance the likelihood of therapeutic targeting of specific lncRNAs.

\section{Acknowledgments}

Financial support and sponsorship:

This study was supported in part by grants from NHLBI (R21 AG060413-01), Welch foundation and Leducq Foundation Trans-Atlantic network of Excellence. 


\section{References and recommended reading}

Papers of particular interest, published within the annual period of review, have been highlighted as: [*] of special interest [**] of outstanding interest

1. Savarese G and Lund LH, Global Public Health Burden of Heart Failure. Card Fail Rev, 2017 3(1): p. 7-11. [PubMed: 28785469]

2. Hangauer MJ, Vaughn IW, and McManus MT, Pervasive transcription of the human genome produces thousands of previously unidentified long intergenic noncoding RNAs. PLoS Genet, 2013 9(6): p. e1003569. [PubMed: 23818866]

3. Mercer TR and Mattick JS, Understanding the regulatory and transcriptional complexity of the genome through the structure. Genome Res, 2013 23(7): p. 1081-8. [PubMed: 23817049]

4. Djebali S, et al., Landscape of transcription in human cells. Nature, 2012 489(7414): p. 101-8. [PubMed: 22955620]

5. Gurha P and Marian AJ, Noncoding RNAs in cardiovascular biology and disease. Circ Res, 2013 113(12): p. e115-20. [PubMed: 24311620]

6. Sallam T, Sandhu J, and Tontonoz P, Long Noncoding RNA Discovery in Cardiovascular Disease: Decoding Form to Function. Circ Res, 2018 122(1): p. 155-166. [PubMed: 29301847]

7. Touma M, et al., Decoding the Long Noncoding RNA During Cardiac Maturation: A Roadmap for Functional Discovery. Circ Cardiovasc Genet, 2016 9(5): p. 395-407. [PubMed: 27591185]

8. Uchida $S$ and Dimmeler S, Long noncoding RNAs in cardiovascular diseases. Circ Res, 2015 116(4): p. 737-50. [PubMed: 25677520]

9. Ma L, Bajic VB, and Zhang Z, On the classification of long non-coding RNAs. RNA Biol, 2013 10(6): p. 925-33. [PubMed: 23696037]

10. St Laurent G, Wahlestedt C, and Kapranov P, The Landscape of long noncoding RNA classification. Trends Genet, 2015 31(5): p. 239-51. [PubMed: 25869999]

11. Boon RA, et al., Long Noncoding RNAs: From Clinical Genetics to Therapeutic Targets? J Am Coll Cardiol, 2016 67(10): p. 1214-1226. [PubMed: 26965544]

12. Thum T, Translational Opportunities and Challenges of Long Noncoding RNAs in Cardiac Regeneration. J Am Coll Cardiol, 2018 72(5): p. 551-552. [PubMed: 29945752]

13. Ounzain S, et al., Discovery and functional characterization of cardiovascular long noncoding RNAs. J Mol Cell Cardiol, 2015 89(Pt A): p. 17-26. [PubMed: 26408097]

14. Philippen LE, et al., Non-coding RNA in control of gene regulatory programs in cardiac development and disease. J Mol Cell Cardiol, 2015 89(Pt A): p. 51-8. [PubMed: 25820097]

15. Fazal FM and Chang HY, lncRNA Structure: Message to the Heart. Mol Cell, 2016 64(1): p. 1-2. [PubMed: 27716479]

16. Han P, et al., A long noncoding RNA protects the heart from pathological hypertrophy. Nature, 2014 514(7520): p. 102-106. [PubMed: 25119045]

17. Ounzain S and Pedrazzini T, The promise of enhancer-associated long noncoding RNAs in cardiac regeneration. Trends Cardiovasc Med, 2015 25(7): p. 592-602. [PubMed: 25753179]

18. Cahill TJ, Choudhury RP, and Riley PR, Heart regeneration and repair after myocardial infarction: translational opportunities for novel therapeutics. Nat Rev Drug Discov, 2017 16(10): p. 699-717. [PubMed: 28729726]

19. Porrello ER, et al., Transient regenerative potential of the neonatal mouse heart. Science, 2011 331(6020): p. 1078-80. [PubMed: 21350179]

20**. Cai B, et al., The Long Noncoding RNA CAREL Controls Cardiac Regeneration. J Am Coll Cardiol, 2018 72(5): p. 534-550. [PubMed: 30056829] This study along with reference 21**, $23 * *$ define the role of IncRNAs in cardiac regeneration by targeting miRNA function.

$21^{* *}$. Chen G, et al., Loss of long non-coding RNA CRRL promotes cardiomyocyte regeneration and improves cardiac repair by functioning as a competing endogenous RNA. J Mol Cell Cardiol, 2018 122: p. 152-164 [PubMed: 30125571]

22. Jain R, et al., HEART DEVELOPMENT. Integration of Bmp and Wnt signaling by Hopx specifies commitment of cardiomyoblasts. Science, 2015 348(6242). 
23**. Li X, et al., Loss of AZIN2 splice variant facilitates endogenous cardiac regeneration. Cardiovasc Res, 2018 114(12): p. 1642-1655. [PubMed: 29584819]

24. Li B, et al., Sirt1 Antisense Long Noncoding RNA Promotes Cardiomyocyte Proliferation by Enhancing the Stability of Sirt1. J Am Heart Assoc, 2018 7(21): p. e009700. [PubMed: 30608184]

25. Chen Y, et al., Long Non-coding RNA ECRAR Triggers Post-natal Myocardial Regeneration by Activating ERK1/2 Signaling. Mol Ther, 2019 27(1): p. 29-45. [PubMed: 30528086]

26. Wang J, et al., A long noncoding RNA NR_045363 controls cardiomyocyte proliferation and cardiac repair. J Mol Cell Cardiol, 2018 127: p. 105-114. [PubMed: 30553885]

$27 * *$. Zhang Y, et al., Long non-coding RNA CCRR controls cardiac conduction via regulating intercellular coupling. Nat Commun, 2018 9(1): p. 4176. [PubMed: 30301979] This is one of the first study implicating lncRNAs in the regulation of cardiac conduction.

28. Nakamura M and Sadoshima J, Mechanisms of physiological and pathological cardiac hypertrophy. Nat Rev Cardiol, 2018 15(7): p. 387-407. [PubMed: 29674714]

29. Heineke J and Molkentin JD, Regulation of cardiac hypertrophy by intracellular signaling pathways. Nat Rev Mol Cell Biol, 2006 7(8): p. 589-600. [PubMed: 16936699]

30. Vervliet T, Robinson EL, and Roderick HL, Lnc'ing Ca(2+), SERCA and cardiac disease. Cell Calcium, 2018 72: p. 132-134. [PubMed: 29793762]

31**. Zhang Y, et al., LncRNA ZFAS1 as a SERCA2a Inhibitor to Cause Intracellular Ca(2+) Overload and Contractile Dysfunction in a Mouse Model of Myocardial Infarction. Circ Res, 2018 122(10): p. 1354-1368. [PubMed: 29475982] The authors describe an important lncRNA function that pertains to its role in regulating cardiac function by maintaining cardiac calcium homeostasis.

32. Latos PA, et al., Airn transcriptional overlap, but not its lncRNA products, induces imprinted Igf2r silencing. Science, 2012 338(6113): p. 1469-72. [PubMed: 23239737]

33. Hosen MR, et al., Airn Regulates Igf2bp2 Translation in Cardiomyocytes. Circ Res, 2018 122(10): p. 1347-1353. [PubMed: 29483092]

34. Simion V, Haemmig S, and Feinberg MW, LncRNAs in vascular biology and disease. Vascul Pharmacol, 2018.

35. Zelcer $\mathrm{N}$ and Tontonoz $\mathrm{P}$, Liver $\mathrm{X}$ receptors as integrators of metabolic and inflammatory signaling. J Clin Invest, 2006 116(3): p. 607-14. [PubMed: 16511593]

36**. Sallam T, et al., Transcriptional regulation of macrophage cholesterol efflux and atherogenesis by a long noncoding RNA. Nat Med, 2018 24(3): p. 304-312. [PubMed: 29431742] In this report, the authors by using several biochemical and molecular approaches define the mechanism of lncRNA (MeXis) function and its role in cholesterol transport.

37. Cremer S, et al., Hematopoietic Deficiency of the Long Non-Coding RNA MALAT1 Promotes Atherosclerosis and Plaque Inflammation. Circulation, 2018.

38. Plaza S, Menschaert G, and Payre F, In Search of Lost Small Peptides. Annu Rev Cell Dev Biol, 2017 33: p. 391-416. [PubMed: 28759257]

39. Pauli A, Valen E, and Schier AF, Identifying (non-)coding RNAs and small peptides: challenges and opportunities. Bioessays, 2015 37(1): p. 103-12. [PubMed: 25345765]

40. Andrews SJ and Rothnagel JA, Emerging evidence for functional peptides encoded by short open reading frames. Nat Rev Genet, 2014 15(3): p. 193-204. [PubMed: 24514441]

41. Nelson BR, et al., A peptide encoded by a transcript annotated as long noncoding RNA enhances SERCA activity in muscle. Science, 2016 351(6270): p. 271-5. [PubMed: 26816378]

42. Anderson DM, et al., A micropeptide encoded by a putative long noncoding RNA regulates muscle performance. Cell, 2015 160(4): p. 595-606. [PubMed: 25640239]

43**. Makarewich CA, et al., The DWORF micropeptide enhances contractility and prevents heart failure in a mouse model of dilated cardiomyopathy. Elife, 2018 7.LncRNA encoded small peptide named as DWORF can rescue HF in a DCM mouse model. 


\section{KEY POINTS}

- Recent discoveries have highlighted the roles of lncRNAs in the regulation of cardiac myocyte proliferation, cardiac conduction, and cholesterol efflux

- $\quad$ LncRNAs regulate cardiac phenotypes by targeting miRNA activity (through direct binding to them) and or by protein through the formation of RNAProtein complex (RNP).

- $\quad$ Future mechanistic studies aimed at defining lncRNA cell type-specific functions will be critical for their therapeutic targeting in the context of Heart failure 